\title{
Extracellular vesicles-released parathyroid hormone-related protein from Lewis lung carcinoma induces lipolysis and adipose tissue browning in cancer cachexia
}

\author{
Wenjun $\mathrm{Hu}^{1}$, Hairong Xiong ${ }^{1}$, Zeyuan $\mathrm{Ru}^{1}$, Yan Zhao ${ }^{1}$, Yali Zhou' ${ }^{1}$, Kairu Xie ${ }^{1}$, Wen Xiao ${ }^{2}$, Zhiyong Xiong ${ }^{2}$,
} Cheng Wang ${ }^{2}$, Changfei Yuan², Jian Shi' ${ }^{2}$ Quansheng Dư ${ }^{3}$, Xiaoping Zhang ${ }^{2}$ and Hongmei Yang $\mathbb{B}^{1}$

\begin{abstract}
Cancer cachexia is a metabolic disorder characterized by skeletal muscle wasting and white adipose tissue browning. Specific functions of several hormones, growth factors, and cytokines derived from tumors can trigger cachexia. Moreover, adipose tissue lipolysis might explain weight loss that occurs owing to cachexia. Extracellular vesicles (EVs) are involved in intercellular communication. However, whether EVs participate in lipolysis induced by cancer cachexia has not been thoroughly investigated. Using Lewis lung carcinoma (LLC) cell culture, we tested whether LLC cellderived EVs can induce lipolysis in 3T3-L1 adipocytes. EVs derived from LLC cells were isolated and characterized biochemically and biophysically. Western blotting and glycerol assay were used to study lipolysis. LLC cell-derived EVs induced lipolysis in vivo and vitro. EVs fused directly with target 3T3-L1 adipocytes and transferred parathyroid hormone-related protein (PTHrP), activating the PKA signaling pathway in 3T3-L1 adipocytes. Blocking PTHrP activity in LLC-EVs using a neutralizing antibody and by knocking down PTHR expression prevented lipolysis in adipocytes. Inhibiting the PKA signaling pathway also prevents the lipolytic effects of EVs. In vivo, suppression of LLC-EVs release by knocking down Rab27A alleviated white adipose tissue browning and lipolysis. Our data showed that LLC cellderived EVs induced adipocyte lipolysis via the extracellular PTHrP-mediated PKA pathway. Our data demonstrate that LLC-EVs induce lipolysis in vitro and vivo by delivering PTHrP, which interacts with PTHR. The lipolytic effect of LLC-EVs was abrogated by PTHR knockdown and treatment with a neutralizing anti-PTHrP antibody. Together, these data show that LLC-EV-induced lipolysis is mediated by extracellular PTHrP. These findings suggest a novel mechanism of lipid droplet loss and identify a potential therapeutic strategy for cancer cachexia.
\end{abstract}

\section{Introduction}

Cancer cachexia, a metabolic disorder, is characterized by skeletal muscle wasting and white adipose tissue

\footnotetext{
Correspondence: Hongmei Yang (hyang@hust.edu.cn)

${ }^{1}$ Department of Pathogenic Biology, School of Basic Medicine, Tongji Medical College, Huazhong University of Science and Technology, Wuhan 430030 Hubei Province, China

${ }^{2}$ Department of Urology, Union Hospital, Tongji Medical College, Huazhong University of Science and Technology, Wuhan 430022 Hubei Province, China Full list of author information is available at the end of the article These authors contributed equally: Wenjun Hu, Hairong Xiong

Edited by A. Stephanou
}

(WAT) browning ${ }^{1}$. Patients with cachexia may lower their food intake, while exhibiting negative energy balance as a result of hypermetabolism, which cannot be improved by nutritional support ${ }^{2}$. This, in turn, might lead to muscle wasting, which is directly correlated with mortality and a lower quality of life; loss of fat mass is also a prognostic marker for poor outcome ${ }^{3,4}$. Although the mechanisms underlying muscle atrophy have been studied widely, much less is known about the factors that initiate the loss of fat in patients with cancer.

\section{(c) The Author(s) 2021}

(c) Open Access This article is licensed under a Creative Commons Attribution 4.0 International License, which permits use, sharing, adaptation, distribution and reproduction in any medium or format, as long as you give appropriate credit to the original author(s) and the source, provide a link to the Creative Commons license, and indicate if changes were made. The images or other third party material in this article are included in the article's Creative Commons license, unless indicated otherwise in a credit line to the material. If material is not included in the article's Creative Commons license and your intended use is not permitted by statutory regulation or exceeds the permitted use, you will need to obtain permission directly from the copyright holder. To view a copy of this license, visit http://creativecommons.org/licenses/by/4.0/. 
Adipose tissues are classified as WAT and brown adipose tissue $(\mathrm{BAT})^{5}$. They often perform opposite physiological functions, with WAT typically contributing to energy accumulation, and BAT contributing to the dissipation of energy as heat. Fat loss is more rapid than lean tissue loss in cancer cachexia. Interestingly, WAT can switch to BAT, and this phenomenon is called 'white adipose browning', which can initiate thermogenesis and induce fat wasting in mice with cancer cachexia ${ }^{5,6}$. Clinical studies suggest that fat depletion in cachexia occurs via lipolysis ${ }^{5,7,8}$. However, the molecular basis of cancerinduced loss of adipose tissue is poorly understood.

Changes in lipid catabolism and lipogenesis drive severe lipid loss in cancer-associated cachexia ${ }^{7,9}$. In particular, glycerol and free fatty acids (FFAs) generated due to hydrolysis of triglycerides, are released into circulation. Indeed, higher levels of FFAs and glycerol are present in the circulation of cachectic patients ${ }^{7,10}$. Both hormonesensitive lipase (HSL) activity and plasma glycerol are increased in patients with cancer cachexia ${ }^{7,10,11}$. Therefore, we sought to determine the mechanisms underlying fat loss in cancer cachexia. Our study was based on a novel hypothesis that the effects of the tumor on adipose tissue are mediated by its released extracellular vesicles (EVs).

EVs are produced by many cell types and can transfer cell contents (e.g. proteins, microRNAs, mRNAs, lipids, or DNA) among different cells. There are three classes of EVs: microvesicles (100-1000 $\mathrm{nm}$ in diameter), apoptotic blebs, and exosomes (30-150 nm). EVs can modulate the biological function of recipient cells by carrying their contents to the cytosol of recipient cells and are key players in tumor progression and drug resistance ${ }^{12-14}$. MicroRNA profiling of circulating tumor exosomes could potentially be used as a surrogate diagnostic procedure and as a noninvasive biomarker for early non-small cell lung cancer diagnosis ${ }^{15,16}$. Exosomes from melanoma cells modify bone marrow progenitor cells towards a prometastatic phenotype via mesenchymal-epithelial transition $^{17}$. Moreover, adrenomedullin containing exosomes secreted by pancreatic cancer cells promote lipolysis in adipose tissue ${ }^{18}$, while tumors can induce muscle atrophy in mice by releasing extracellular heat shock proteins (Hsp70 and Hsp90) ${ }^{19}$. Therefore, we hypothesized that tumor-promoting EVs may play a role in the crosstalk between tumor cells and adipocytes, which leads to lipolysis and energy expenditure, and subsequently to fat loss in cancer cachexia.

Parathyroid hormone-related protein (PTHrP) can stimulate thermogenic gene expression by binding to parathyroid hormone receptor (PTHR, which is known to be highly expressed in kidneys, bone, adipose, and muscle tissues ${ }^{20}$ ), which is shared by parathyroid hormone (PTH) and $\mathrm{PTHrP}^{20}$. PTHrP induces lipolysis via protein kinase
A (PKA)-mediated phosphorylation of $\mathrm{HSL}^{20,21}$. Additionally, bone-derived PTHrP and adiponectin are involved in whole-body metabolism, which is regulated by bone-adipose hormonal relay ${ }^{22}$. Moreover, PTHrP derived from Lewis lung carcinoma (LLC) tumors triggers adipose tissue browning with increased energy production via activation of uncoupling protein 1 (UCP1) and cancer cachexia via binding to PTHR. Interestingly, neutralizing antibodies of PTHrP restrains browning, the loss of adipose depots, and muscle wasting in models of cancer cachexia $^{21}$. Moreover, PTHrP can inhibit adipocyte differentiation by downregulating PPAR $\gamma$ activity via a MAPK-dependent mechanism ${ }^{23}$. Additionally, PTHrP can be secreted by numerous tumors and is related to hypercalcemia of malignancy associated with cancer cachexia $^{24-26}$. Therefore, we proposed that PTHrP in EVs could serve as a mediator of lipolysis and browning in cancer cachexia.

The conditioned medium from LLC cells (LCM) activates a catabolic response in cultured 3T3-L1 adipocytes that demonstrate lipids metabolism in LLC tumor-bearing mice $^{21,27}$. This finding suggests that LLC cells release cachexins that directly induce lipolysis and adipose tissue browning. Additionally, we previously found that cachexia-inducing tumor cells release high levels of EVs that serve as carriers of tumor-released PTHrP. However, the role of EVs in lipolysis and the effectors utilized in the process are as of yet unknown.

In the present study, we found that lipolysis occurs in adipocytes upon exposure to LLC-derived EVs (LLCEVs). To confirm the role of PTHrP, we blocked PTHrP activity in LLC-EVs by using a neutralizing antibody. Furthermore, we assessed WAT browning and fat loss in mice implanted with LLC cells in which the expression of Rab27A (a GTPase that controls different steps of EV release) had been stably knocked down with shRNA.

\section{Materials and methods \\ Reagents}

Recombinant mouse PTHrP was purchased from Wuhan USCN Business Co. Ltd. (Wuhan, China). We purchased anti-PTHrP (1-34) antibody (T-4512) from Peninsula Laboratories International (San Carlos, CA, USA). H89 (ab143787), the inhibitor of PKA, and antiUCP1 (ab10983) were purchased from Abcam (Cambridge, MA, USA). All other antibodies were purchased from Cell Signaling Technology (Danvers, MA, USA).

\section{LLC cell culture and animals}

LLC cells were maintained in Dulbecco's modified Eagle's medium (DMEM) (Invitrogen, Carlsbad, CA, USA) with $10 \%$ fetal bovine serum, $1 \%$ penicillin, and streptomycin. C57BL/6 mice from Beijing HFK Bioscience (Beijing, China) were used for all experiments. We used 6- 
to -10-week-old male mice in all experiments. The mice were randomly divided into four groups (each group has nine mice, $n=9$ ): mice injected with PBS, mice injected with LLC cells, mice injected with LLC cells transduced with lentiviral shRab27A, and mice injected with LLC cells transduced with lentiviral control. Cells $\left(5 \times 10^{6}\right)$ were injected subcutaneously into the flanks of the mice. Mice were euthanized on day 21 following tumor implantation. Body weight was then determined and iWAT and eWAT were immediately harvested and weighed. For subsequent studies, the iWAT and eWAT were fixed in $4 \%$ paraformaldehyde and the other tissues were immediately frozen in liquid nitrogen and stored at $-80^{\circ} \mathrm{C}$.

\section{Lipid droplet areas}

We chose 3 representative images of each section from three individual mice to quantify the lipid droplet areas using ImageJ software (National Institutes of Health, Bethesda, MD). The detailed protocol has been described previously ${ }^{27}$.

\section{Isolation of LLC-EVs}

EV extraction was performed according to previously published protocols ${ }^{18}$. Briefly, cells were grown to $\sim 70 \%$ confluence and cultured in 10\% exosome-free fetal bovine serum (System Biosciences, Palo Alto, CA, USA) for $72 \mathrm{~h}$. We collected the culture medium, which we subjected to centrifugation at $3000 \mathrm{rpm}$ for $10 \mathrm{~min}$ at $4{ }^{\circ} \mathrm{C}$ to remove debris. We then subjected the supernatant to centrifugation at $100,000 \times g$ for $60 \mathrm{~min}$ using an ultracentrifuge (Beckman, Brea, CA, USA) to pellet the EVs. EVs were then washed in PBS and subjected to centrifugation at $100,000 \times g$ for $60 \mathrm{~min}$. The resulting pellets were resuspended in PBS. We assessed the protein concentrations in the EVs with a BCA Protein Assay Kit (Thermo Fisher, Waltham, MA, USA).

\section{T3-L1 cell culture and differentiation}

Murine 3T3-L1 cells were obtained from the American Type Culture Collection (Manassas, VA, USA) and cultured in DMEM with $10 \%$ fetal calf serum. Confluent 3T3-L1 cells were treated with a cocktail of $0.5 \mathrm{mM}$ isobutylmethylxanthine, $1 \mathrm{mM}$ dexamethasone, $5 \mathrm{mg} / \mathrm{ml}$ insulin, and $5 \mathrm{mM}$ troglitazone (all from Sigma-Aldrich, St Louis, MO, USA) to induce differentiation. After 2 days of differentiation, the cells were maintained in a medium with insulin until harvest. Experiments were conducted using differentiated adipocytes (10-14 days).

\section{Oil red $\mathrm{O}$ staining of adipocytes}

Adipocytes were stained with Oil red $\mathrm{O}$ solution (Goodbio Technology, Wuhan, China). Briefly, cells were washed three times with PBS and fixed in $4 \%$ formalin.
After fixation, we diluted $0.5 \%$ Oil red $\mathrm{O}$ in isopropanol (Sigma-Aldrich) with water (3:2), filtered the solution through a $0.45-\mu \mathrm{m}$ filter, and incubated with the fixed cells for $1 \mathrm{~h}$ at room temperature. After staining, images were obtained using a Leica DMI 3000B microscope (Wetzlar, Germany).

\section{Glycerol estimation assay}

Differentiated adipocytes were serum-starved overnight in DMEM and $2 \%$ bovine serum albumin (fatty acid-free,) then treated with exosome-free DMEM (phenol red-free) with $0.5 \%$ bovine serum albumin for $24 \mathrm{~h}$. We assayed the supernatants for glycerol levels with free glycerol reagent (Sigma-Aldrich), as per the manufacturer's recommendations.

\section{Internalization of labeled EVs}

EVs were labeled with $2 \mu \mathrm{M}$ PKH67 (Sigma-Aldrich) for $4 \mathrm{~min}$, washed, then incubated for $12 \mathrm{~h}$ with cultured 3T3-L1 adipocytes ( 3000 cells/well). Cells were washed, counterstained with 2-(4-amidinophenyl)- $1 \mathrm{H}$-indole-6carboxamidine, and imaged under an Olympus FV500 fluorescence microscope (Tokyo, Japan).

\section{Electron microscopy}

EVs were fixed in $1 \%$ glutaraldehyde in PBS (pH 7.4). After rinsing, a 30- $\mu \mathrm{l}$ drop of the suspension was loaded onto a formvar/carbon-coated grid, negatively stained with $3 \%(\mathrm{w} / \mathrm{v})$ aqueous phosphotungstic acid for $90 \mathrm{~s}$, and observed by transmission electron microscopy (JEM 1200EX, Jeol, Tokyo, Japan).

\section{Nanoparticle analysis}

We analyzed the EV fractions on a Malvern instrument (Malvern Instruments, Malvern, UK). The EV particles were counted and analyzed using the Nanoparticle Tracking Analysis software.

\section{Western blotting}

Western blotting was performed as previously described $^{27}$. Briefly, proteins from adipose tissue, differentiated 3T3-L1 cells, and EVs were extracted using radioimmunoprecipitation assay protein lysis buffer (P1003; Beyotime Institute of Biotechnology, Shanghai, China) with freshly added $1 \%$ protease inhibitor cocktail and $1 \mathrm{mM}$ phenylmethylsulphonyl fluoride. Each well was loaded with $50 \mu \mathrm{g}$ protein and incubated overnight at $4{ }^{\circ} \mathrm{C}$ with primary antibodies. We purchased antibodies phospho-PKA substrate (catalog number 9624), phosphoHSL (catalog number 4126), and HSL (catalog number 4107) from Cell Signaling Technology; antibodies against UCP1(catalog number ab10983), tubulin (catalog number ab18207), CD9 (catalog number ab92726), TSG101 (catalog number 125011), and Hsp70 (catalog number 2787) 
from Abcam; and an antibody against Rab27A from Proteintech (Wuhan, China). We performed quantitative analyses of protein levels with ImageJ software.

\section{Immunofluorescent staining}

3T3-L1 adipocytes were seeded and differentiated on chamber slides. The cells were washed with PBS, fixed in $4 \%$ paraformaldehyde, permeabilized with $0.3 \%$ Triton $^{\mathrm{Tm}}$ $\mathrm{X}-100$ for $15 \mathrm{~min}$, and blocked with 3\% bovine serum albumin for $30 \mathrm{~min}$. Cells were then incubated with antibodies against PTHrP and PTHR, followed by Alexa Fluor $^{\circledR}$ 488- or Alexa Fluor ${ }^{\circledR}$ 594-conjugated secondary antibody. Nuclei were stained with 2-(4-amidinophenyl)$1 \mathrm{H}$-indole-6-carboxamidine. Images were taken with an Olympus FV500 fluorescence microscope (Tokyo, Japan).

\section{shRNA expression in LLC and 3T3-L1 cells}

For the shRNA-mediated knockdown of Rab27A and PTHR, we purchased lentiviral vectors encoding shRNAs from GeneChem (Shanghai, China) and packaged them in viral particles according to the manufacturer's recommendations. Mouse Rab27A shRNA sense sequences used were:5' - AGTACACTGATGGCAAGTT-3'; PTHR sense sequences used were:5'-CCAGCCATTGAGAACGA-AA3'; LLC cells transduced with shRNA targeting Rab27A and 3T3-L1 preadipocytes transduced with shRNA targeting PTHR were selected by puromycin.

\section{Statistical analyses}

Data are presented as the mean \pm standard error of the mean. We performed one-way analysis of variance followed by Bonferroni's post hoc correction to compare the conditions in both cell and animal experiments. Differences were deemed significant when the $p$ value was $<0.05,\left({ }^{*} P<0.05,{ }^{* * *} P<0.01,{ }^{* * * *} P<0.001,{ }^{* * * * * *} P<0.0001\right)$.

\section{Results}

\section{Characterization of LLC-EVs}

We isolated LLC-EVs from serum-free culture medium through multistep centrifugation ${ }^{18}$. Analysis of LLC-EVs by electron microscopy revealed the cup-shaped vesicles (Fig. 1A). We quantitatively characterized the mean LLCEV diameter as $109 \mathrm{~nm}$ using a Zetasizer Nano (Fig. 1B). Furthermore, we detected the exosomal markers Hsp70, TSG101, and CD9 in LLC-EVs and colon-26 (C26)derived EVs (C26-EVs; C26 that also induce cachexia in mice) by western blotting analysis (Fig. 1C). Then, we labeled the EVs with PKH67, incubated with 3T3-L1 adipocytes, and observed green fluorescence in the adipocytes by confocal fluorescence microscopy (Fig. 1D). However, the internalization of LLC-EVs into adipocytes could be inhibited by nystatin $(54 \mu \mathrm{M}$, an inhibitor of caveolin/lipid raft-mediated endocytosis); While, chlorpromazine $(20 \mu \mathrm{M}$, an inhibitor of clathrin-mediated endocytic pathway), amiloride $(50 \mu \mathrm{M}$, an inhibitor of micropinocytosis), and nocodazole ( $1 \mu \mathrm{M}$, a destabilizer of microtubule) did not exhibit present the analogous effect. This finding suggested that adipocytes take up EVs through caveolin/lipid raft-mediated endocytosis.

\section{LLC-EVs induced lipolysis in vitro}

To explore the effect of LLC-EVs on lipid metabolism, we incubated 3T3-L1 adipocytes with LCM (LLC-cellconditioned medium), which contains EVs, and EVdepleted LCM (LCM-dep). We measured lipolysis by assessing glycerol release and by western blotting for phosphorylated HSL (a marker of lipolysis) and the level of UCP1 (uncoupling protein 1; a mitochondrial protein expressed in multilocular cells that can uncouple oxidative phosphorylation and produce heat). Western blotting of 3T3-L1 adipocytes demonstrated that LCM activated lipolysis, whereas the effect of LCM-dep was comparatively weak (Fig. 2A). In the glycerol assay, the degree of glycerol release was higher in response to LCM than LCM-dep (Fig. 2B). These findings suggested that LLCEVs may be involved in lipolysis.

To further explore the role of LLC-EVs in lipolysis, we disrupted tumor cell release of EVs by knocking down the expression of Rab27A $\mathrm{A}^{28,29}$ (Fig. 2C). Subsequently, we quantified the EVs in the supernatants of LLC cell cultures by assessing the activity of acetylcholinesterase $(A c h E)^{30}$. Treatment with LCM from cells exposed to Rab27A shRNA inhibited the activity of AchE compared to treatment with the LCM from cells treated with the control lentiviral vector (Fig. 2D). Furthermore, western blotting suggested that the ratio of phospho-HSL: HSL and the expression of UCP1 were lower in adipocytes incubated with LCM from cells exposed to Rab27A shRNA than in those treated with the control LCM (Fig. $2 \mathrm{E})$. In addition, glycerol release from adipocytes incubated with LCM from cells exposed to Rab27A shRNA was lower than from those treated with control LCM (Fig. 2F). These findings further confirmed that LLC-released EVs contribute to lipolysis.

\section{PTHrP contained in LLC-EVs was involved in lipolysis in 3T3-L1 adipocytes}

PTHrP released by LLC cells into LCM promotes thermogenic gene expression in adipocytes. Interestingly, we observed that PTHrP can be detected in LLC-EVs obviously, but not clearly in C26-EVs. Furthermore. Subsequently, we assessed the levels of PTHrP in the supernatant of various tumor cells capable of triggering cachexia, including mouse lung carcinoma cell (LLC) and C26 colon carcinoma cells (C26). We detected higher levels of PTHrP in the supernatant of LLC than in that of C26 by enzyme-linked immunosorbent assay (Fig. 3A). Therefore, the constitutive release of $\mathrm{PTHrP}$ is not a 
A
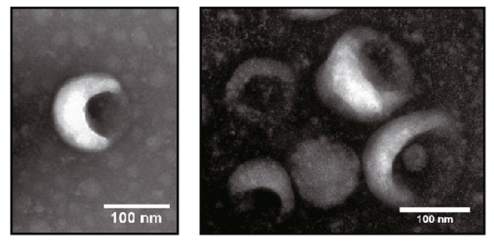

D
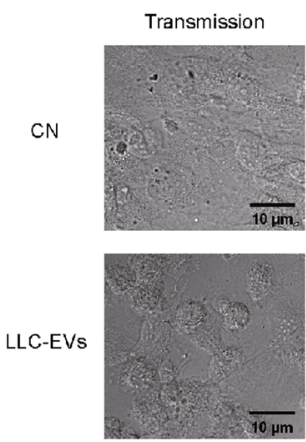

DAPI
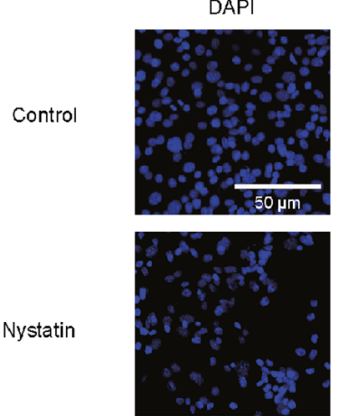

Nystatin

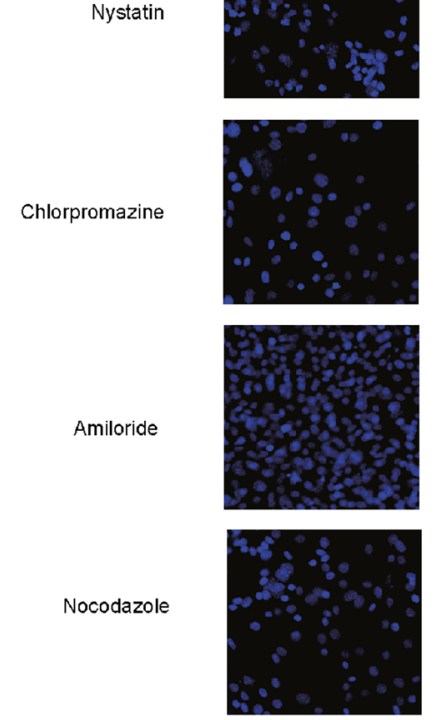

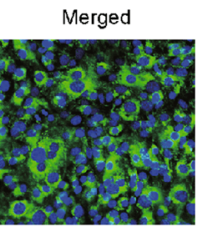
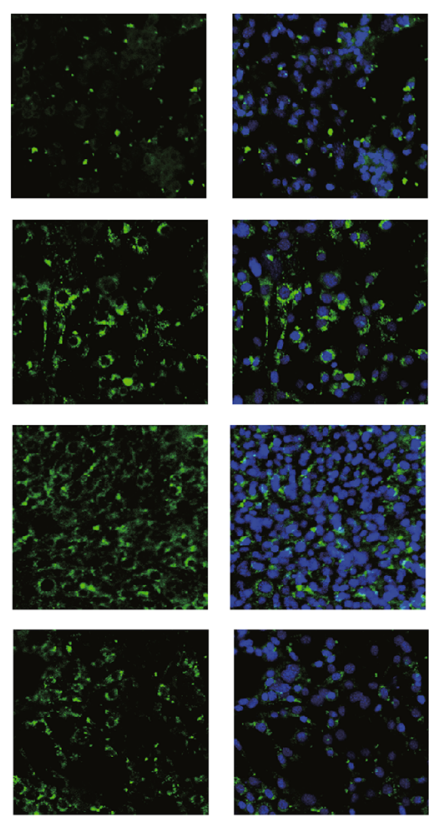
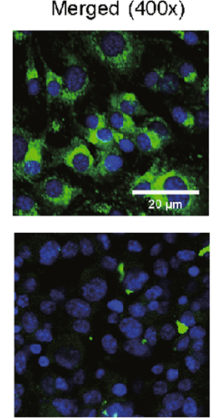

C

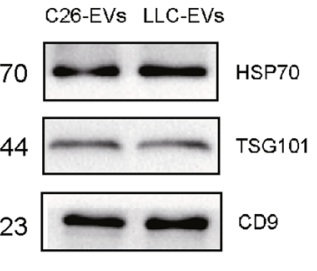

Merge
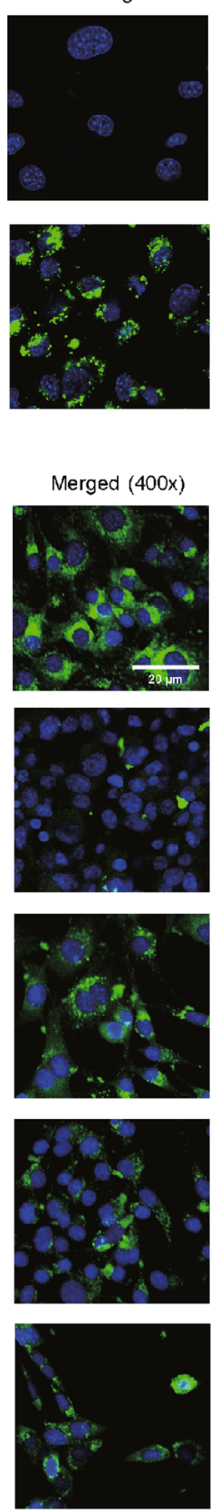

Fig. 1 Analysis of LLC-EV characteristics. A Analysis of EVs by electron microscopy (bars $=100 \mathrm{~nm}$ ). B Size of LLC-EVs isolated from LLC cells (mean diameter: $109 \mathrm{~nm})$. C Western blotting analysis of the expression of Hsp70, TSG101, and CD9 in EVs isolated from C26 and LLC cells. D Confocal images (20X) of differentiated adipocytes pretreated with various inhibitors of endocytic pathways and incubated with labeled LLC-EVs for overnight at $37^{\circ} \mathrm{C}$. Amiloride $(50 \mu \mathrm{M} ; 30 \mathrm{~min})$, nystatin $(54 \mu \mathrm{M} ; 30 \mathrm{~min})$, chlorpromazine $(20 \mu \mathrm{M} ; 30 \mathrm{~min})$, and nocodazole $(1 \mu \mathrm{M} ; 10 \mathrm{~min})$. 

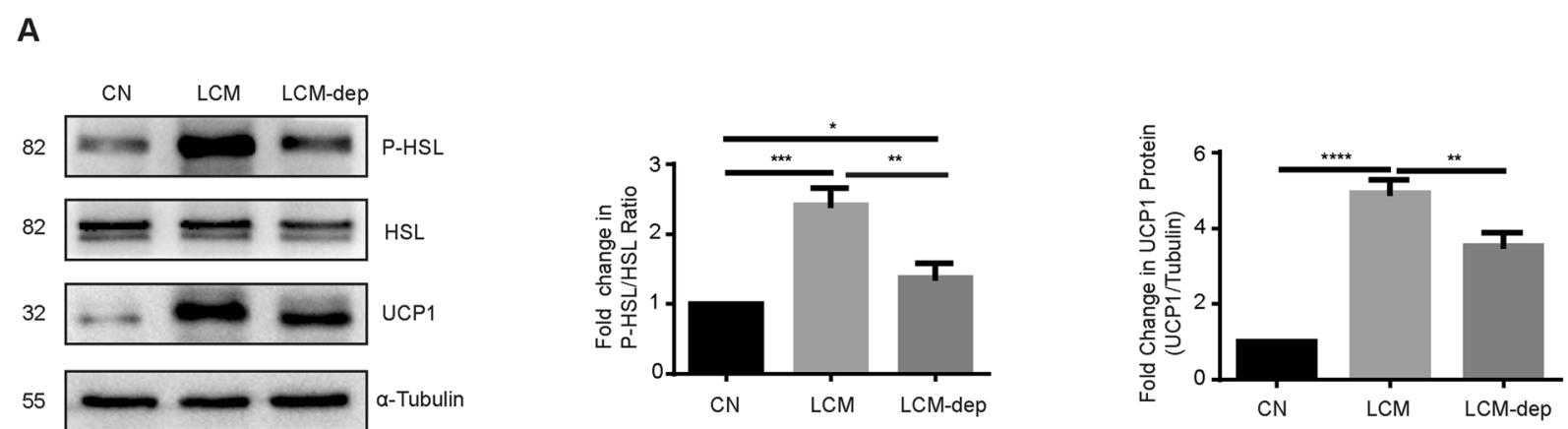

B

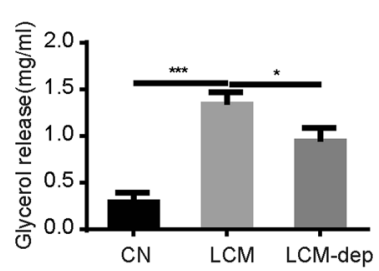

C

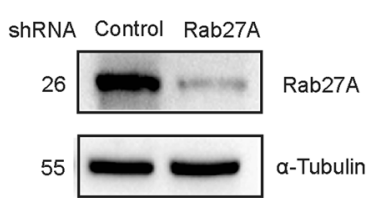

D

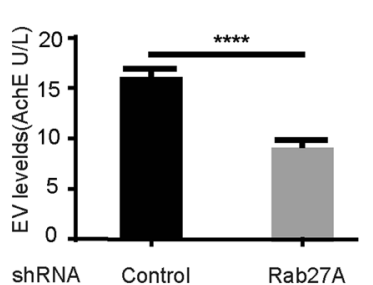

ShRNA

Control

Rab27A

\section{E}
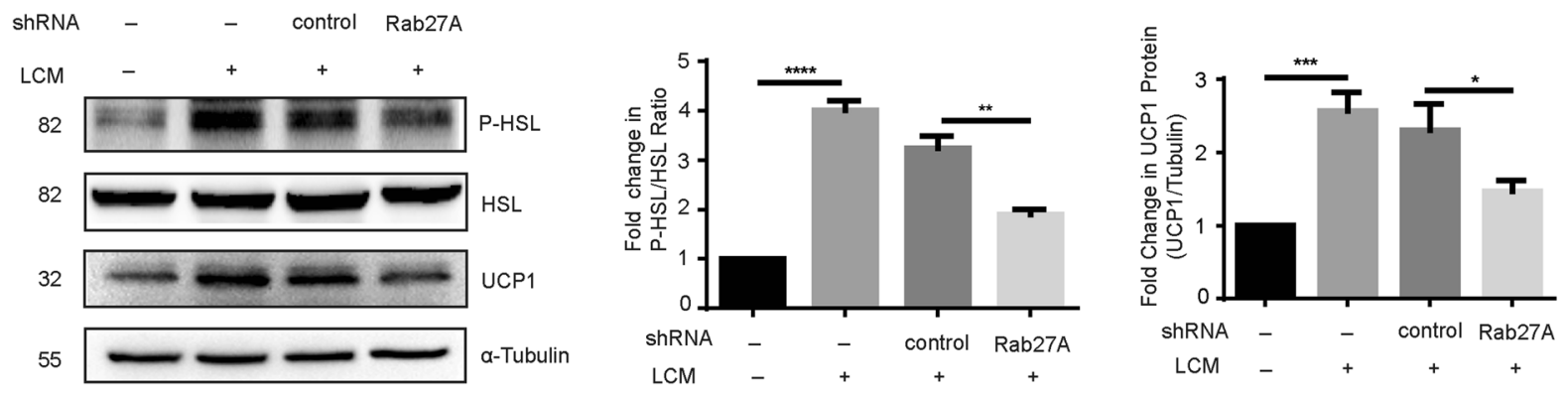

Fig. 2 LLC-EVs have a lipolytic action in 3T3-L1 adipocytes. A Western blotting showing the relative level of phospho-HSL: HSL and UCP1 in 3T3L1 adipocytes. Data ( $n=3$ ) were analyzed by analysis of variance (ANOVA). B Glycerol levels in the supernatants of 3T3-L1 adipocytes incubated with LCM or LCM-dep. Data $(n=3)$ were analyzed by analysis of variance (ANOVA). C Western blotting showing the level of Rab27A in LLC cells. D AchE levels in supernatants of LLC cell transfected with shRNA-Rab27A or control lentivirus vector. Data $(n=4)$ were analyzed by analysis of variance (ANOVA). E Western blotting showing the relative level of phospho-HSL: HSL and UCP1 in 3T3-L1 adipocytes incubated with or without LCM, and LCM (Rab27A shRNA or control shRNA). Data $(n=8)$ were analyzed by analysis of variance (ANOVA). F Glycerol levels in the supernatants of 3T3-L1 adipocytes incubated with or without LCM, and LCM (Rab27A shRNA or control shRNA). Data $(n=3)$ were analyzed by analysis of variance (ANOVA). ${ }^{*} P<0.05,{ }^{* *} P<0.01,{ }^{* * *} P<0.001,{ }^{* * *} P<0.0001$, ns: not significant.

common characteristic shared by different types of cancer cells that can induce cancer cachexia. Furthermore, utilizing patient data from the database associated with the web-based Kaplan-Meier plotter KmPlot, we divided patients into two groups based on the mRNA expression levels in their tumor biopsies. We observed a significantly shorter overall survival for patients with lung cancer with PTHrP mRNA levels above the median value (hazard ratio $=1.25$ [1.1-1.42], logrank $P=0.00048, n=2,437$; Fig. 3B). To assess the effect of PTHrP on lipid metabolism, we treated 3T3-L1 adipocytes with various doses of
PTHrP (100 ng/ml and $200 \mathrm{ng} / \mathrm{ml})$ and immunoblotted to detect their expression of phospho-HSL, HSL, and UCP1. The ratio of phospho-HSL: HSL and level of UCP1 expression were significantly higher in adipocytes treated with PTHrP (Fig. 3C). Additionally, we observed fewer lipid droplets in 3T3-L1 adipocytes treated with PTHrP $(100 \mathrm{ng} / \mathrm{ml})$ and higher levels of glycerol in the supernatants after PTHrP treatment compared with those in the control group (Fig. 3D, E); TNF- $\alpha$ was used as a positive control. Next, western blotting demonstrated that the ratio of phospho-HSL: HSL and level of UCP1 


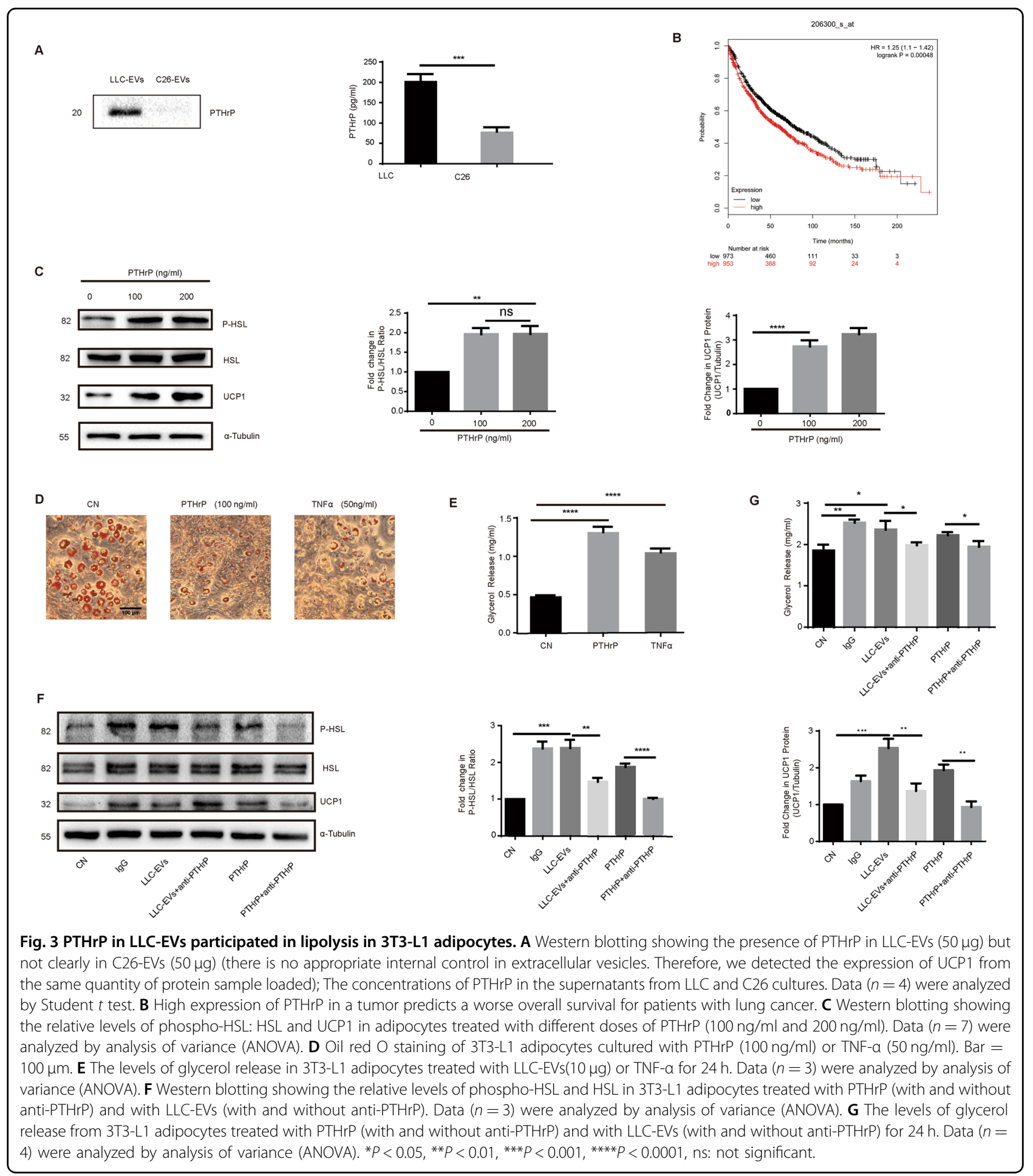

expression increased upon treatment with PTHrP and LLC-EVs, whereas treatment with anti-PTHrP antibody neutralized this effect (Fig. 3F). Furthermore, we assessed glycerol release in response to PTHrP in cell supernatants and LLC-EVs in the presence and absence of an anti-
PTHrP neutralizing antibody. Notably, the anti-PTHrP antibody alleviated lipolysis induced by PTHrP and LLCEVs (Fig. 3G). These findings further confirmed that the induction of lipolysis by LLC-EVs is partially dependent on extracellular PTHrP. 


\section{PTHR is critical to LLC tumor-induced lipolysis}

PTHR-mediated wasting in cachexia occurs through a common mechanism involving PTHR. Hence, we investigated if lipolysis was induced by PTHrP and LLC-EVs through interaction with PTHR by transfecting 3T3-L1 adipocytes with shRNA targeting PTHR, PTHR expression had been stably knocked down (Fig. 4A). Confocal localization showed more PTHrP/PTHR interactions, characterized by yellow punctate dots formed by merged red and green punctate dots, in the presence of increasing amounts of LLC-EVs. However, LLC-EVs treatment of adipocytes treated with PTHR shRNA abolished the receptor-ligand interactions (Fig. 4B). The lipolysis induced by PTHrP and LLC-EVs as assessed by glycerol release was also suppressed by treatment with shRNA targeting PTHrP (Fig. 4C, D). Furthermore, we observed that the effects of PTHrP and LLC-EVs on the ratio of phospho-HSL: HSL and UCP1 required PTHR (Fig. 4E, F), suggesting that LLC-EVs and PTHrP induce lipid droplet lipolysis via interaction with PTHR on 3T3-L1 adipocytes. Similarly, thus, our results demonstrate that 3T3-L1 adipocytes lacking PTHR are resistant to lipolysis driven by tumor cell-released EVs.

\section{Lipolytic effect of extracellular PTHrP was mediated through the PKA pathway}

PTHrP has been shown to interact with the G proteincoupled PTHR, which stimulates the phosphorylation of the PKA substrates $\mathrm{HSL}^{20}$. Hence, we investigated if extracellular PTHrP-induced lipolysis through the activation of PKA in adipocytes by examining the effects of H89, a selective PKA inhibitor. Western blotting showed that the PKA pathway activation and increased HSL phosphorylation in adipocytes induced by $\mathrm{PTHrP}$ were suppressed by H89 (Fig. 5A). Similarly, the PTHrPinduced increase in glycerol release by 3T3-L1 adipocytes was suppressed by H89 (Fig. 5B). Interestingly, western blotting revealed that the effects of LLC-EVs on PKA pathway activation and HSL phosphorylation in adipocytes were suppressed by H89 (Fig. 5C), as was their effect on glycerol release (Fig. 5D). These findings suggested that tumor cells released extracellular PTHrP-induced lipolysis by activating PKA-mediated lipid catabolism.

\section{Tumor-released PTHrP-expressing EVs caused lipolysis and WAT browning}

To determine whether tumor cell-released PTHrPexpressing EVs are critical for adipose tissue lipolysis and browning, we disrupted LLC release of EVs in vivo by knocking down the expression of Rab27A before tumor implantation. We observed that mice implanted with LLC cells in which Rab27A expression had been stably knocked down did not experience abnormal decreases in free body, inguinal WAT (iWAT), or epididymal WAT
(eWAT) weight, or increases in serum glycerol release, serum AchE activity, or PTHrP levels (Fig. 6A-F). Furthermore, western blotting showed that the ratio of phospho-HSL: HSL and the expression of UCP1 were suppressed in eWAT and iWAT of mice implanted with LLC cells lacking Rab27A (Fig. 6G, H). Similarly, hematoxylin and eosin revealed significantly smaller adipocytes with multilocular cytoplasm in both the eWAT and iWAT of mice implanted with LLC cells lacking Rab27A compared to not lacking Rab27A (Fig. 6I); UCP1 staining revealed significantly that the expression of UCP1 is lower in both the eWAT and iWAT of mice implanted with LLC cells lacking Rab27A compared to not lacking Rab27A (Fig. 6J). These findings suggested that targeting tumor cell-released PTHrP-expressing EVs could be an effective therapeutic strategy for cancer cachexia.

\section{Discussion}

Our study provides a novel insight into the function of tumor-derived EVs. We found that LLC-EVs fuse with and promote the lipolysis of adipocytes in vitro and in vivo via activation of PKA signaling. Importantly, our data suggest that tumor-released extracellular PTHrP is a key cachexin responsible for adipose loss in tumorbearing mice.

PTHrP is secreted by many tumors and has a known role in cancer cachexia. It affects lipolysis and inhibits adipocyte differentiation ${ }^{20,23}$. In our study, we observed that both recombinant and extracellular PTHrPpromoted lipolysis through the activation of the PKA pathway; this effect was abrogated in the presence of an anti-PTHrP antibody. However, in our C26-induced cachexia model, we also observed WAT browning and fat droplet loss in the presence weakly of PTHrP containing C26-EVs. The phenomenon demonstrated that different types of tumor-derived EVs carry different cargoes and may mediate different pathways of internalization into recipient cells ${ }^{31,32}$. This suggests that different tumors induce similar phenotypes via different mechanisms that must be targeted by distinct therapeutic modalities.

Our data demonstrate that LLC-EVs induce lipolysis in vitro, which is abrogated by PTHR knockdown, suggesting that LLC-EV-induced lipolysis is partially dependent on extracellular PTHrP. We also showed that LLCEVs do not contain functionally relevant levels of TNF- $\alpha$, a well-known lipolytic factor (Figure S1). We speculate that other factors may contribute to LLC-induced lipolysis either independently or in conjunction with LLC-derived extracellular PTHrP. Additionally, to explore the distribution of tumor-derived EVs in vivo, we injected PKH67 labeled EVs into the tail veins of mice. In subcutaneous adipose tissue and epididymal adipose tissue, we could observe the presence of labeled EVs (Figure S2). However, 


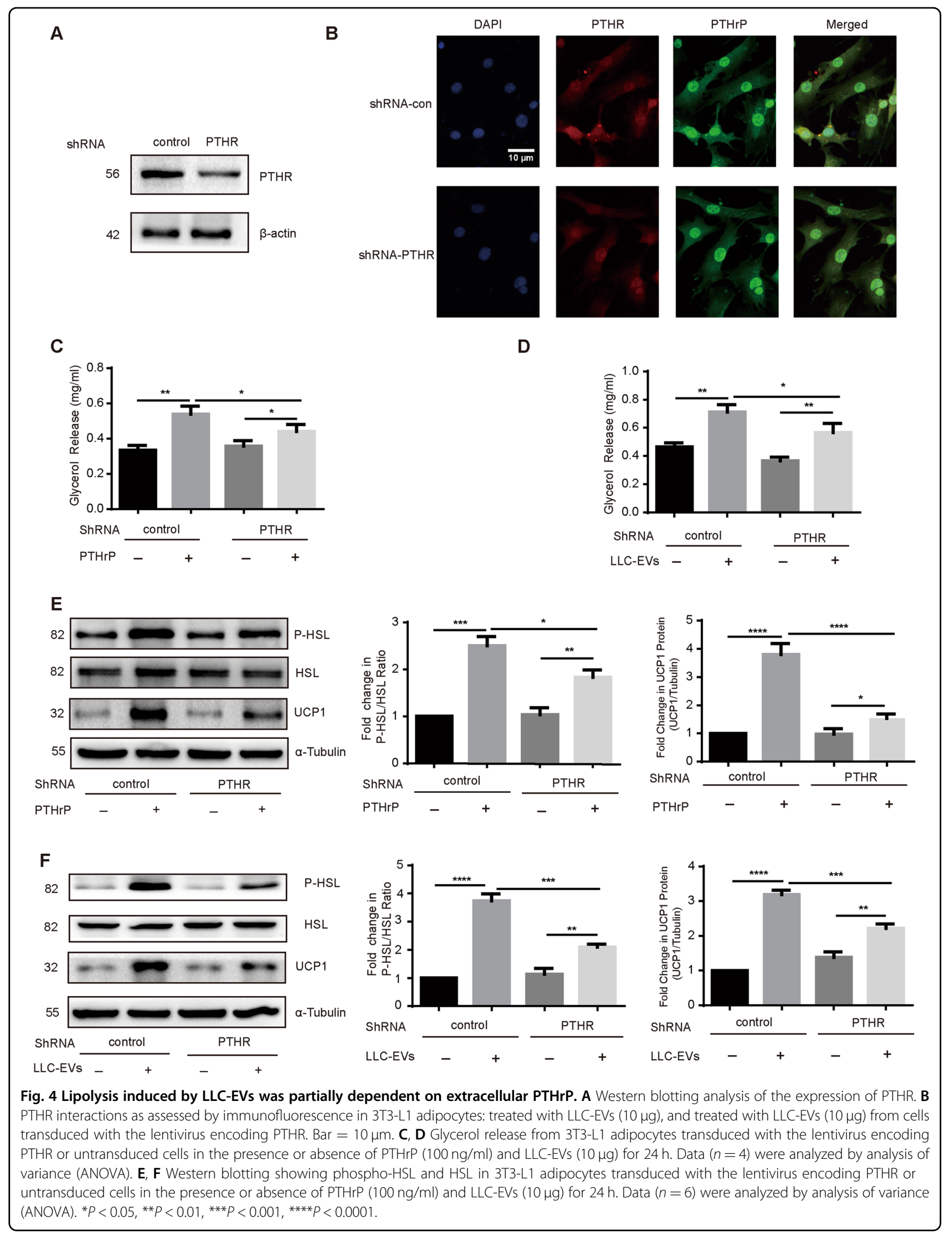



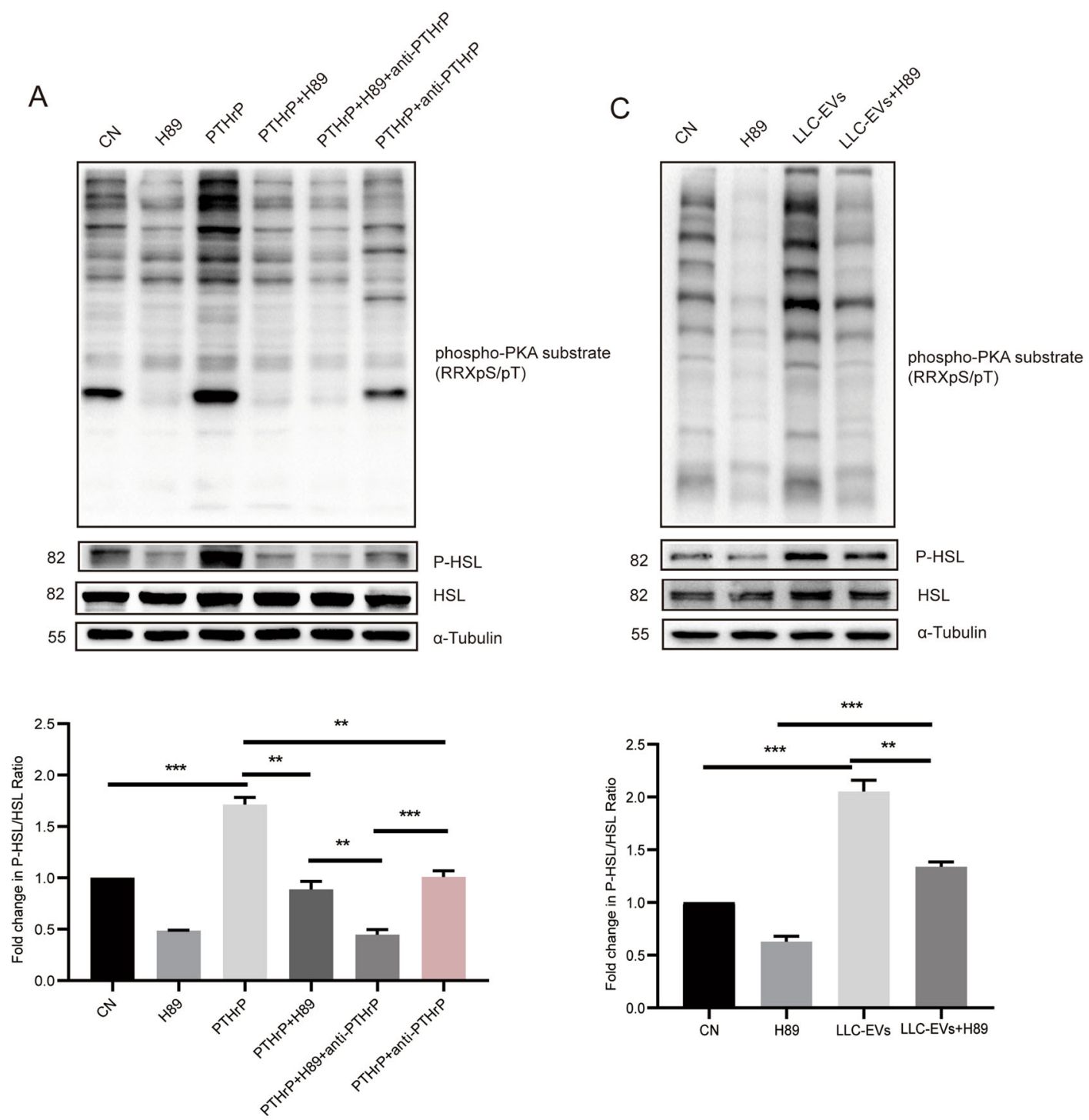

B

$\mathrm{D}$
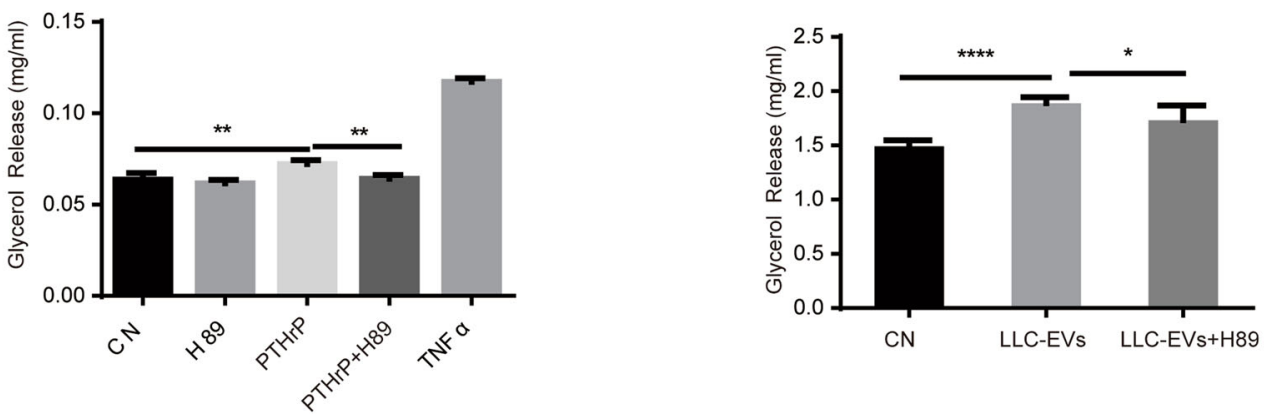

Fig. 5 The lipolytic effect of extracellular PTHrP was mediated through the PKA pathway. 3T3-L1 adipocytes were serum-starved for $2 \mathrm{~h}$ and pretreated with $50 \mu \mathrm{M} \mathrm{H} 89$ for $1 \mathrm{~h}$, then PTHrP $(100 \mathrm{ng} / \mathrm{ml})$ was added for $30 \mathrm{~min}$ to analyze protein phosphorylation by western blotting (A) or for $24 \mathrm{~h}$ to assess glycerol release (B). 3T3-L1 adipocytes were serum-starved for $2 \mathrm{~h}$ and pretreated with $50 \mu \mathrm{M} \mathrm{H} 89 \mathrm{for} 1 \mathrm{~h}$, then LLC-EVs (10 $\mu \mathrm{g})$ were added for 30 min to analyze protein phosphorylation by western blotting $(\mathbf{C})$ or for $24 \mathrm{~h}$ to assess glycerol release (D). Data $(n=3)$ were analyzed by analysis of variance (ANOVA). ${ }^{*} P<0.05,{ }^{* *} P<0.01,{ }^{* *} P<0.001,{ }^{* * *} P<0.0001$. 


$$
\text { A }
$$

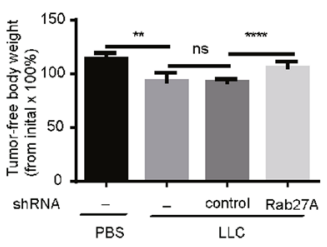

D

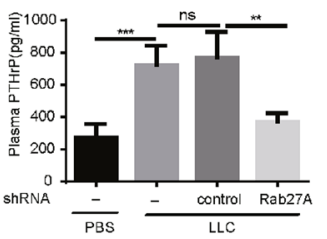

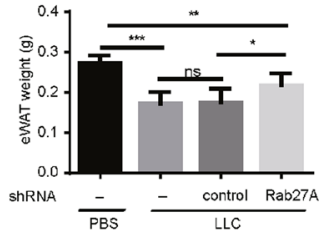

E

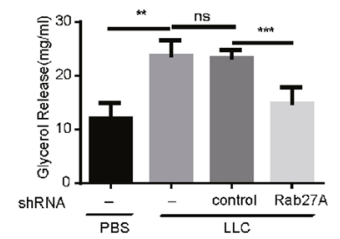

C

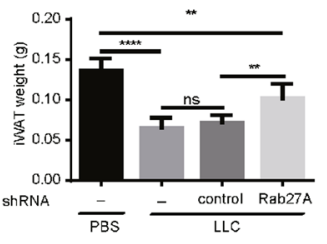

F

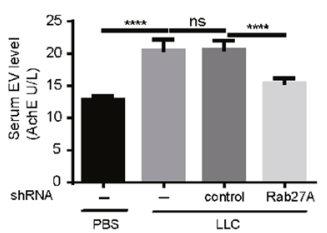

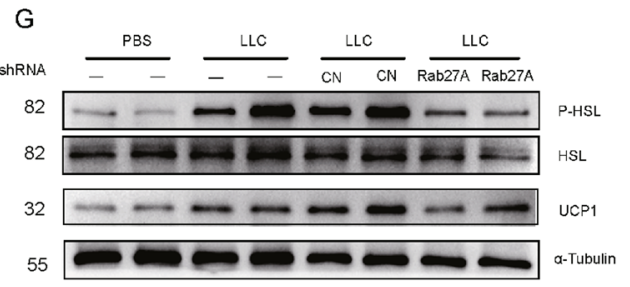
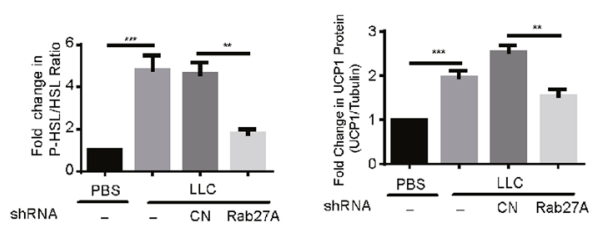

$\mathrm{H}$
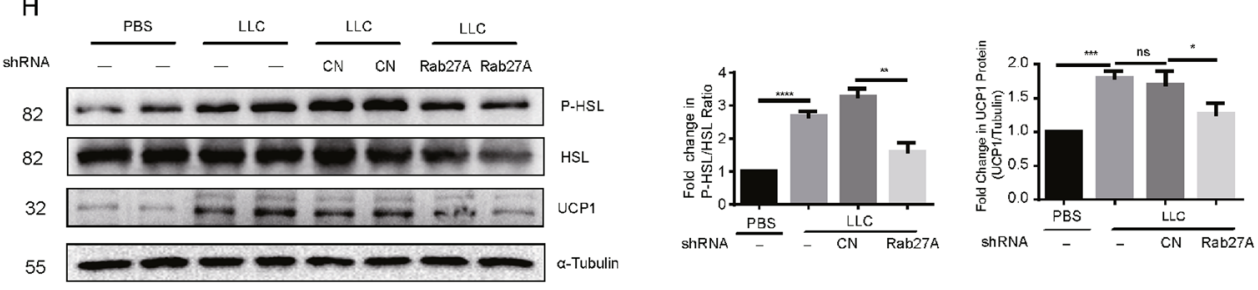

shRNA
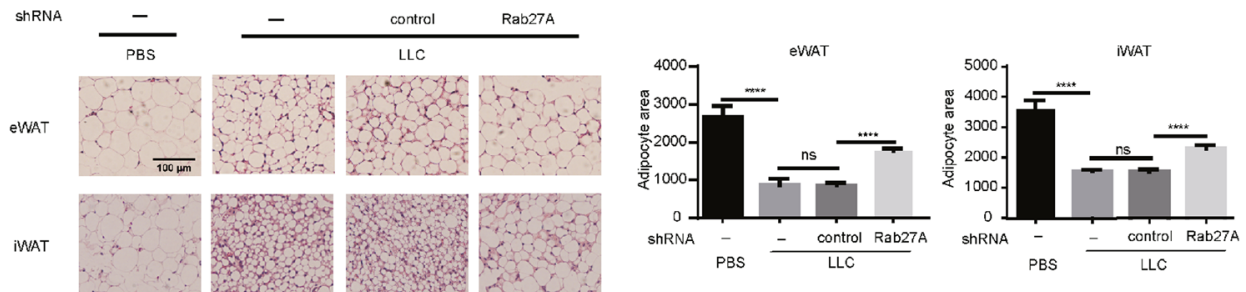

J

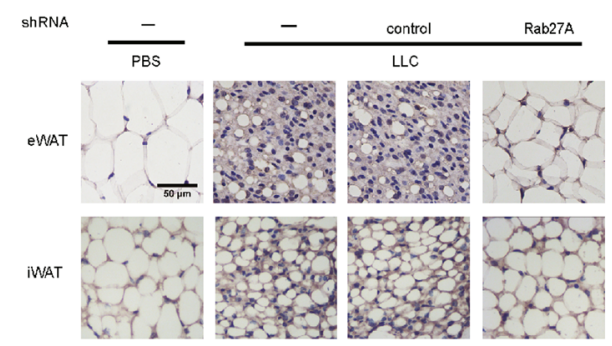

Fig. 6 Tumor-released PTHrP-expressing EVs caused lipolysis and WAT browning ( $\boldsymbol{n}=\mathbf{9}$ for each group). (A) Weight change of the tumor-free body. Data $(n=3)$ were analyzed by analysis of variance (ANOVA) (B, C) Weight change of iWAT and eWAT. Data $(n=3)$ were analyzed by analysis of variance (ANOVA) (D, E, F) Level of PTHrP concentration, glycerol release, and serum AchE activity in the serum of mice. Data $(n=3)$ were analyzed by analysis of variance (ANOVA) $(\mathbf{G}, \mathbf{H})$ western blotting analysis the level of P-HSL and UCP1 in eWAT and iWAT. Data $(n=3)$ were analyzed by analysis of variance (ANOVA) $(\mathbf{I}, \mathbf{J})$ Hematoxylin and eosin and UCP1 staining of eWAT and iWAT. Data $(n=3)$ were analyzed by analysis of variance (ANOVA). ${ }^{*} P<0.05,{ }^{* *} P<0.01,{ }^{* * *} P<0.001,{ }^{* * *} P<0.0001$, ns: not significant. 
the effects of LLC-EVs in vivo and the mechanism behind lipolysis induced by LLC-EVs in vivo and vitro will be further explored in our future studies.

EVs can provide therapeutic opportunities for the treatment of various types of tumors ${ }^{33}$. We detected tumor-derived EVs in supernatants of LLC that were similar to exosomes in terms of size $(\sim 109 \mathrm{~nm})$ and protein expression (Hsp70/TSG101/CD9). We used the term 'extracellular vesicles' for these vesicles to conform to the recommendations of the International Society for Extracellular Vesicles ${ }^{34}$. Targeting Rab27B can inhibit exosome-mediated transfer miR-34c-5p and increase its intracellular level; this research provides a new strategy for the treatment of patients with acute myeloid leuke$\mathrm{mia}^{35}$. Inhibiting exosome generation in sepsis by GW4869 can suppress the sepsis triggered inflammatory response and then improve cardiac function and survi$\mathrm{val}^{36}$. Similarly, inhibiting cancer cell-derived EVs release and biogenesis by GW4869 can change the EV emission profiles reflective of drug-related therapeutic stress and thereby, EV-based assays can serve as companion diagnostics for targeted anticancer agents ${ }^{37}$. Additionally, EVs released by cancer cells can be used as effective carriers of paclitaxel to their parental cells, carrying the drug into cells and increasing its cytotoxicity ${ }^{38,39}$. Furthermore, EVs can be used as therapeutics and as diagnostic biomarkers in clinical application ${ }^{40,41}$. In the cancer cachexia model, inhibiting LLC-EVs release by knocking down the expression of Rab27A and Rab27B can alleviate muscle wasting $^{19}$. In our study, inhibiting LLC-EVs release by knocking down Rab27A expression could relieve fat loss and WAT browning in cancer cachectic mice. However, in patients with cancer, how to reasonably utilize EVs in clinical therapeutics for cachexia still requires more effort and further research.

In our study, we showed that tumor-released EVs mediate lipolysis of lipid droplets through the cargo protein PTHrP. Kri et al. previously reported that LLC tumors released a high level of PTHrP, which induced WAT browning and fat loss ${ }^{21}$. However, Zhang et al. reported that PTHrP levels released into culture supernatants by LLC cells were similar to those released by nontumorigenic cells and that serum PTHrP levels in mice with cachexia induced by LLC cells were comparable to those in controls ${ }^{19}$. In our study, we observed that serum PTHrP levels are higher in cachectic mice than in control mice. Furthermore, serum PTHrP concentrations in mice bearing LLC transduced with shRNA against Rab27A are lower than those in mice injected with LLC transduced with the empty vector. In addition, we detected PTHrP in EVs released by LLC cells but not in EVs released by C26 cells, which also induced cachexia with WAT browning. Therefore, we conclude that different LLC cell subtypes release distinct cachexins and EVs with different contents.
We observed that recombinant PTHrP and extracellular PTHrP exert their effects by interacting with PTHR (encoded by Pth1r) on adipocytes. Previous reports have also demonstrated that PTHrP promotes lipolysis by binding to PTHR and activating the PKA pathway ${ }^{20,42}$. Similarly, we found that PTHrP and LLC-EVs activate the PKA pathway and lipolysis, which can be partially inhibited by H89. This finding further confirmed that LLC-EVs contain PTHrP. Based on these observations, we propose that tumor-released EVs induce WAT browning and lipid droplet loss by activating the PKA/CREB/HSL catabolic signaling pathway. Furthermore, lung tumor exosomes can suppress adipogenesis of human mesenchymal stem cells through TGF $\beta$ signaling pathway ${ }^{43}$. Therefore, we hypothesize that the effects of reduced adipogenesis and increased lipolysis induced by tumor-released EVs, may contribute to fat loss and WAT browning in cancer cachexia. This hypothesis and possible mechanisms require further research. By packaging specific cargo, LLC-EVs appear to function as important mediators of paracrine intercellular communication and may involve specific pathways for internalization in recipient cells (including adipocytes), depending on their cargo, size, and cell signaling status ${ }^{31}$. In our study, we observed caveolin/ lipid raft-mediated endocytosis as a possible mechanism for EVs internalization in adipocytes. However, other mechanisms and molecules mediating EVs internalization into recipient cells still require exploration to provide a strategy for clinical therapy.

Our data demonstrate that LLC-EVs induce lipolysis in vitro and in vivo by delivering PTHrP, which interacts with PTHR. The lipolytic effect of LLC-EVs can be abrogated by PTHR knockdown and treatment with a neutralizing anti-PTHrP antibody. Together, these data show that LLC-EV-induced lipolysis is mediated by extracellular PTHrP. These findings suggest a novel mechanism of lipid droplet loss and identify a potential therapeutic strategy for cancer cachexia.

\section{Acknowledgements \\ This study was partly supported by Union Hospital, Tongji Medical College, Huazhong University of Science and Technology and Department of Urology.}

\section{Funding}

This research was supported by the National Natural Science Foundation of China (NSFC) Grant [81773282 and 81672528] and Wuhan Science and Technology Plan Application Foundation Frontier Project (2020020601012247).

\section{Author details}

${ }^{1}$ Department of Pathogenic Biology, School of Basic Medicine, Tongji Medical College, Huazhong University of Science and Technology, Wuhan 430030 Hubei Province, China. ${ }^{2}$ Department of Urology, Union Hospital, Tongji Medical College, Huazhong University of Science and Technology, Wuhan 430022 Hubei Province, China. ${ }^{3}$ Department of Neuroscience and Regenerative Medicine, Medical College of Georgia, Augusta University, Augusta, GA 30912, USA 


\section{Author contributions}

Wenjun Hu designed the study and drafted the manuscript, carried out the experiments, and analyzed the figures. Hairong Xiong carried out the experiments and analyzed the figures. Zeyuan Ru prepared the experiment and contributed to get acquisition of data. Yan Zhao and Yali Zhou contributed to the conception of the experiments and check the interpretation of data. Kairu Xie contributed to get acquisition of data. Wen Xiao and Zhiyong Xiong helped to design experiment, analysis, and interpretation of data. Cheng Wang, Changfei Yuan, and Jian Shi contributed to the analysis of data and interpretation of data. Quansheng Du and Xiaoping Zhang revise the final approval of the version to be published and perform with constructive advice of the manuscript. Hongmei Yang contributed to conceptions, design the studies, and revise final approval of the version to be published.

\section{Data availability}

All data analyzed during this study are included in this published article.

\section{Conflict of interest}

The authors declare that they have no conflict of interest.

\section{Ethics approval}

Ethics approval was obtained from the Animal Care and Use Committee of Tongji Medical College of Huazhong University of Science and Technology (approval no. S2392).

\section{Publisher's note}

Springer Nature remains neutral with regard to jurisdictional claims in published maps and institutional affiliations.

Supplementary Information accompanies this paper at (https://doi.org/ 10.1038/s41419-020-03382-0).

Received: 1 March 2020 Revised: 5 December 2020 Accepted: 21 December 2020

Published online: 28 January 2021

\section{References}

1. Argiles, J. M., Busquets, S., Stemmler, B. \& Lopez-Soriano, F. J. Cancer cachexia: understanding the molecular basis. Nat. Rev. Cancer 14, 754-762 (2014).

2. Barile, L. \& Vassalli, G. Exosomes: therapy delivery tools and biomarkers of diseases. Pharmacol. Ther. 174, 63-78 (2017).

3. Becker, A. et al. Extracellular vesicles in cancer: cell-to-cell mediators of metastasis. Cancer cell 30, 836-848 (2016).

4. Bobrie, A. et al. Rab27a supports exosome-dependent and -independent mechanisms that modify the tumor microenvironment and can promote tumor progression. Cancer Res. 72, 4920-4930 (2012).

5. Chan, G. K., Deckelbaum, R. A., Bolivar, l., Goltzman, D. \& Karaplis, A. C. PTHrP inhibits adipocyte differentiation by down-regulating PPAR gamma activity via a MAPK-dependent pathway. Endocrinology 142, 4900-4909 (2001).

6. Clancy, J. \& D'Souza-Schorey, C. Extracellular vesicles in cancer: purpose and promise. Cancer J. 24, 65-69 (2018).

7. Essandoh, K. et al. Blockade of exosome generation with GW4869 dampens the sepsis-induced inflammation and cardiac dysfunction. Biochim. Biophys. Acta 1852, 2362-2371 (2015).

8. Fearon, K. C., Glass, D. J. \& Guttridge, D. C. Cancer cachexia: mediators, signaling, and metabolic pathways. Cell Metab. 16, 153-166 (2012).

9. Fouladiun, M. et al. Body composition and time course changes in regional distribution of fat and lean tissue in unselected cancer patients on palliative care-correlations with food intake, metabolism, exercise capacity, and hormones. Cancer 103, 2189-2198 (2005).

10. Gyorgy, B., Hung, M. E., Breakefield, X. O. \& Leonard, J. N. Therapeutic applications of extracellular vesicles: clinical promise and open questions. Annu. Rev. Pharmacol. Toxicol. 55, 439-464 (2015).

11. Iguchi, H., Onuma, E., Sato, K., Sato, K. \& Ogata, E. Involvement of parathyroid hormone-related protein in experimental cachexia induced by a human lung cancer-derived cell line established from a bone metastasis specimen. Int. J. cancer 94, 24-27 (2001)
12. Jin, $X$. et al. Evaluation of tumor-derived exosomal miRNA as potential diagnostic biomarkers for early-stage non-small cell lung cancer using nextgeneration sequencing. Clin. Cancer Res. 23, 5311-5319 (2017).

13. Kir, S. et al. Tumour-derived PTH-related protein triggers adipose tissue browning and cancer cachexia. Nature 513, 100-104 (2014).

14. Kir, S. et al. PTH/PTHrP receptor mediates cachexia in models of kidney failure and cancer. Cell Metab. 23, 315-323 (2016).

15. Lotvall, J. et al. Minimal experimental requirements for definition of extracellular vesicles and their functions: a position statement from the International Society for Extracellular Vesicles. J. Extracell. Vesicles 3, 26913 (2014).

16. Matsumoto, Y. et al. Quantification of plasma exosome is a potential prognostic marker for esophageal squamous cell carcinoma. Oncol. Rep. 36, 2535-2543 (2016)

17. Montermini, L. et al. Inhibition of oncogenic epidermal growth factor receptor kinase triggers release of exosome-like extracellular vesicles and impacts their phosphoprotein and DNA content. J. Biol. Chem. 290, 24534-24546 (2015).

18. Mundy, G. R. \& Edwards, J. R. PTH-related peptide (PTHrP) in hypercalcemia. J. Am. Soc. Nephrol. 19, 672-675 (2008).

19. Murphy, R. A. et al. Loss of adipose tissue and plasma phospholipids: relationship to survival in advanced cancer patients. Clin. Nutr. 29, 482-487 (2010).

20. Ostrowski, M. et al. Rab27a and Rab27b control different steps of the exosome secretion pathway. Nat. Cell Biol. 12, 19-30 (2010). sup pp 1-13.

21. Ovesen, L., Allingstrup, L., Hannibal, J., Mortensen, E. L. \& Hansen, O. P. Effect of dietary counseling on food intake, body weight, response rate, survival, and quality of life in cancer patients undergoing chemotherapy: a prospective, randomized study. J. Clin. Oncol. 11, 2043-2049 (1993).

22. Peinado, $\mathrm{H}$. et al. Melanoma exosomes educate bone marrow progenitor cells toward a pro-metastatic phenotype through MET. Nat. Med. 18, 883-891 (2012).

23. Peng, D. et al. miR-34c-5p promotes eradication of acute myeloid leukemia stem cells by inducing senescence through selective RAB27B targeting to inhibit exosome shedding. Leukemia 32, 1180-1188 (2018).

24. Petruzzelli, M. et al. A switch from white to brown fat increases energy expenditure in cancer-associated cachexia. Cell Metab. 20, 433-447 (2014).

25. Pitt, J. M., Kroemer, G. \& Zitvogel, L. Extracellular vesicles: masters of intercellular communication and potential clinical interventions. J. Clin. Invest. 126, 1139-1143 (2016).

26. Ryden, M. et al. Lipolysis-not inflammation, cell death, or lipogenesis-is involved in adipose tissue loss in cancer cachexia. Cancer 113, 1695-1704 (2008).

27. Saari, H. et al. Microvesicle- and exosome-mediated drug delivery enhances the cytotoxicity of Paclitaxel in autologous prostate cancer cells. J. Control. Release 220, 727-737 (2015).

28. Sagar, G. et al. Pathogenesis of pancreatic cancer exosome-induced lipolysis in adipose tissue. Gut 65, 1165-1174 (2016).

29. Samuel, P., Fabbri, M. \& Carter, D. R. F. Mechanisms of drug resistance in cancer: the role of extracellular vesicles. Proteomics 17, 23-24 (2017).

30. Sun, R. et al. Valproic acid attenuates skeletal muscle wasting by inhibiting C/ EBPbeta-regulated atrogin1 expression in cancer cachexia. Am. J. Physiol. Cell Physiol. 311, C101-C115 (2016).

31. Taylor, D. D. \& Gercel-Taylor, C. MicroRNA signatures of tumor-derived exosomes as diagnostic biomarkers of ovarian cancer. Gynecol. Oncol. 110, 13-21 (2008).

32. Thomas, S. S. \& Mitch, W. E. Parathyroid hormone stimulates adipose tissue browning: a pathway to muscle wasting. Curr. Opin. Clin. Nutr. Metab. Care 20, 153-157 (2017).

33. Tian, T. et al. Exosome uptake through clathrin-mediated endocytosis and macropinocytosis and mediating miR-21 delivery. J. Biol. Chem. 289, 22258-22267 (2014).

34. Tisdale, M. J. Biology of cachexia. J. Natl Cancer Inst. 89, 1763-1773 (1997).

35. Tkach, M. \& Thery, C. Communication by extracellular vesicles: where we are and where we need to go. Cell 164, 1226-1232 (2016).

36. Tsoli, M., Swarbrick, M. M. \& Robertson, G. R. Lipolytic and thermogenic depletion of adipose tissue in cancer cachexia. Semin. Cell Dev. Biol. 54, 68-81 (2016).

37. Walkley, C. R., Walia, M. K., Ho, P. W. \& Martin, T. J. PTHrP, its receptor, and protein kinase A activation in osteosarcoma. Mol. Cell. Oncol. 1, e965624 (2014).

38. Wang, S., Li, X., Xu, M., Wang, J. \& Zhao, R. C. Reduced adipogenesis after lung tumor exosomes priming in human mesenchymal stem cells via TGFbeta signaling pathway. Mol. Cell. Biochem. 435, 59-66 (2017). 
39. Wu, K., Xing, F., Wu, S. Y. \& Watabe, K. Extracellular vesicles as emerging targets in cancer: recent development from bench to bedside. Biochim. Biophys. Acta Rev. Cancer 1868, 538-563 (2017).

40. Zhang, $\mathrm{G}$. et al. Tumor induces muscle wasting in mice through releasing extracellular Hsp70 and Hsp90. Nat. Commun. 8, 589 (2017).

41. Zhang, X., Cheng, Q., Wang, Y., Leung, P. S. \& Mak, K. K. Hedgehog signaling in bone regulates whole-body energy metabolism through a bone-adipose endocrine relay mediated by PTHrP and adiponectin. Cell Death Differ. 24, 225-237 (2017)

42. Zimmermann, R. et al. Fat mobilization in adipose tissue is promoted by adipose triglyceride lipase. Science 306, 1383-1386 (2004).

43. Zuijdgeest-van Leeuwen, S. D. et al. Lipolysis and lipid oxidation in weight-losing cancer patients and healthy subjects. Metabolism 49, 931-936 (2000). 